\title{
Isoflavonoids and Triterpenoids Isolated from Pterodon
}

\author{
polygalaeflorus \\ Délcio D. Marques ${ }^{a}$, Maria Iracema Lacerda Machado ${ }^{a}$, Mário Geraldo de \\ Carvalho $^{b}$, Luiz Augusto da C. Meleira ${ }^{b}$, and Raimundo Braz-Filho ${ }^{c}$ \\ ${ }^{a}$ Departamento de Química Orgânica e Inorgânica, Universidade Federal do Ceará, \\ C.P. 12.200, 60.455-760 Fortaleza - Ce, Brazil \\ ${ }^{b}$ Departamento de Química - ICE, Universidade Federal Rural do Rio de Janeiro, \\ 23851-970 Soropédica-Itaguaí - RJ, Brazil \\ ${ }^{c}$ Setor de Produtos Naturais, Universidade Estadual do Norte Fluminense, \\ 28015-620 Campos - RJ, Brazil
}

Received: April 30, 1997

\begin{abstract}
Os isoflavonóides 6,7-dimetoxi-3',4'-metilenodioxi-, 4'-hidroxi-3',6,7-trimetoxi-, 3,4,6,7tetrametoxi-, 7-hidroxi-6-metoxi-3,4-metilenodioxi-, 2',6,7-trimetoxi-3',4'-metilenodioxi-, 2',3',4',7,7-pentametoxi- e 2',4',5,6,7-pentametoxiisoflavona, os triterpenóides lupeol e betulina e o ácido 4-metoxibenzóico foram isolados dos extratos acetônicos do alburno e do cerne de Pterodon polygalaeflorus. As estruturas destes produtos naturais foram caracterizadas por métodos espectrométricos, principalmente experiências de RMN 1D e 2D de hidrogênio e carbono-13, que foram também utilizados para a atribuição inequívoca dos deslocamentos químicos dos átomos de hidrogênio e carbono-13 dos isoflavonóides.

The isoflavonoids 6,7-dimethoxy-3',4'-methylenodioxy-, 4'-hydroxy-3',6,7-trimethoxy-, 3,4,6,7-tetramethoxy-, 7-hydroxy-6-methoxy-3,4-methylenodioxy-, 2',6,7-trimethoxy-3',4' methylenedioxy-, 2',3',4',6,7-pentamethoxy- and 2',4',5,6,7-pentamethoxyisoflavone, together with the triterpenoids lupeol and betulin and 4-methoxybenzoic acid, were isolated from acetone extracts of the sapwood and heartwood of Pterodon polygalaeflorus. The structures of these natural products have been characterized by spectrometric methods, mainly extensive 1D and 2D NMR experiments, which were also used for complete assignments of the chemical shifts of the hydrogen and carbon-13 atoms of isoflavonoids.
\end{abstract}

Keywords: Pterodon polygalaeflorus, Leguminosae, isoflavonoids, triterpenoids, ${ }^{1} H$ - and ${ }^{13} C-N M R$ data

\section{Introduction}

In a paper published recently the isolation of diterpenes from fruits of a specimen of Pterodon ploygalaeflorus Benth, Leguminosae family, was reported ${ }^{1}$. As part of our continuing chemical investigation of this plant we have investigated the sapwood and the heartwood. The isoflavones 1-7, p-methoxybenzoic acid (8), lupeol (9), and betulin (10), have been isolated from this plant material. The structures of these natural products were established by spectral data, mainly ${ }^{1} \mathrm{H}$ - and ${ }^{13} \mathrm{C}-\mathrm{NMR}$ including ho- monuclear and heteronuclear $2 \mathrm{D}$ experiments $(\mathbf{2}, \mathbf{3 a}, \mathbf{5}$ and 7) and NOE difference spectra (1, 2, 3a, 5 and 7), which were also used for complete assignment of the hydrogen and carbon-13 atom chemical shifts of the isoflavonoid 2 , the acetyl derivatives $\mathbf{3 a}, \mathbf{5}$ and $\mathbf{7}$.

\section{Results and Discussion}

The acetone extracts of heartwood and sapwood of Pterodon ploygalaeflorus were submitted to chromatography on silica gel column to afford the isoflavonoids 1-7, 4-methoxybenzoic acid (8), lupeol (2) and betulin (10). The 
isoflavones 1, 2, 5-7 were previously isolated from Milletia dura $(\mathbf{1} \text { and } \mathbf{5})^{2}$, Pterodon pubescens $(\mathbf{2}, \mathbf{5} \text { and } \mathbf{7})^{3,4}$ and Condyla africana $(\mathbf{6})^{5}$. The triterpenoids 9 and $\mathbf{1 0}$ are frequently found in plants ${ }^{6}$. The structural characterization of these compounds was based on spectral data, notebly the ${ }^{1} \mathrm{H}$ - and ${ }^{13} \mathrm{C}-\mathrm{NMR}$ spectra, including homonuclear ${ }^{1} \mathrm{H}-\mathrm{x}$ ${ }^{1} \mathrm{H}-\mathrm{COSY}$ and heteronuclear ${ }^{1} \mathrm{H}-\mathrm{X}{ }^{13} \mathrm{C}-\mathrm{COSY}-{ }^{1} \mathrm{~J}_{\mathrm{CH}}$ and ${ }^{1} \mathrm{H}-$ $\mathrm{x}{ }^{13} \mathrm{C}-\mathrm{COSY}-{ }^{n} \mathrm{~J}_{\mathrm{CH}}(\mathrm{n}=2$ and 3)] experiments and NOE difference spectra $\left({ }^{1} \mathrm{H}\left\{{ }^{1} \mathrm{H}\right\}-\mathrm{NOE}\right)$ data for the isoflavonoids $1,2,3 \mathbf{a}, 5$ and 7 , in addition to corresponding ${ }^{1} \mathrm{H}-\mathrm{NMR}$ spectral data reported in the literature ${ }^{2-5}$ (Table 2). To the best of our knowledge, the isoflavone 3 is hitherto unreported as a natural product and ${ }^{13} \mathrm{C}$-NMR spectral data were only found for $\mathbf{2}$ (Table 2$)^{7}$.

The homonuclear ${ }^{1} \mathrm{H}-\mathrm{x}{ }^{1} \mathrm{H}-\mathrm{COSY}$, heteronuclear ${ }^{1} \mathrm{H}-\mathrm{x}$ ${ }^{13} \mathrm{C}-\mathrm{COSY}-{ }^{\mathrm{n}}{ }_{\mathrm{JCH}}\left(\mathrm{n}=1\right.$, spin-spin couplings between ${ }^{13} \mathrm{C}$ and ${ }^{1} \mathrm{H}$ - atoms via one bond; $\mathrm{n}=2$ and 3, COLOC $=$ correlation via long-range couplings) $2 \mathrm{D}$ shift-correlated NMR spectra ${ }^{8}$ of 2, 3a, 5 and 7 (Tables 1-3) and NOE difference spectra ${ }^{8}$ of $\mathbf{1}, \mathbf{2}, \mathbf{3 a}, \mathbf{5}$ and $\mathbf{7}$ (Table 4) together with the application of the shift parameters and the observed multiplicities of the signals of the carbon atoms deduced by comparative analysis of the PND- and DEPT${ }^{13} \mathrm{C}$-NMR spectra ${ }^{9}$, were also used for the complete assignment of the hydrogen and carbon-13 atom chemical shifts

Table 1. ${ }^{13} \mathrm{C}-\mathrm{NMR}(50.3 \mathrm{MHz})$ data for the isoflavonoids $\mathbf{1 - 3 , 5}$ and $\mathbf{7}\left(\mathrm{CDCl}_{3}\right)$, compared values described in the literature are in parenthesis ${ }^{7}$ for $\mathbf{2}$, TMS as internal standard and chemical shifts in $\delta(\mathrm{ppm})$.* $^{*}$

\begin{tabular}{|c|c|c|c|c|c|}
\hline $\mathbf{C}$ & 1 & $2^{\mathrm{a}}$ & $3 \mathrm{a}^{\mathrm{a}}$ & $5^{\mathrm{a}}$ & $7^{\mathrm{a}}$ \\
\hline 3 & 124.45 & $123.91(123.33)$ & 123.86 & 124.27 & 121.54 \\
\hline 4 & 175.38 & $175.21(174.57)$ & 175.14 & 175.76 & 175.24 \\
\hline 6 & 147.74 & $147.36(147.56)$ & 147.68 & 147.29 & 147.30 \\
\hline 7 & 154.41 & $154.02(154.36)$ & 154.35 & 153.33 & 153.98 \\
\hline 9 & 152.23 & $151.89(151.93)$ & 152.12 & 152.27 & 152.05 \\
\hline 10 & 117.81 & $117.51(117.29)$ & 117.72 & 117.08 & 117.55 \\
\hline $1^{\prime}$ & 125.85 & $124.51(124.91)$ & 130.88 & 112.50 & 118.49 \\
\hline $2^{\prime}$ & - & - & - & 152.59 & 151.75 \\
\hline $3^{\prime}$ & 147.56 & $148.35(148.80)$ & 150.70 & - & 142.06 \\
\hline 4, & 147.74 & $148.65(148.50)$ & 139.51 & 148.10 & 153.74 \\
\hline 5 , & - & - & - & 140.70 & - \\
\hline \multicolumn{6}{|l|}{ CH } \\
\hline 2 & 151.96 & $151.77(152.84)$ & 152.49 & 153.96 & 153.00 \\
\hline 5 & 104.90 & $104.35(104.42)$ & 104.60 & 104.08 & 104.68 \\
\hline 8 & 99.50 & $99.18(100.05)$ & 99.41 & 99.24 & 99.32 \\
\hline $2^{\prime}$ & 109.72 & $112.13(112.95)$ & 113.55 & - & - \\
\hline 3 & - & - & - & 94.79 & - \\
\hline 5 , & 108.29 & $110.75(111.52)$ & 122.80 & - & 106.96 \\
\hline 6 & 122.33 & $120.61(121.21)$ & 120.66 & 110.53 & 125.63 \\
\hline \multicolumn{6}{|l|}{$\mathrm{CH}_{2}$} \\
\hline $\mathrm{OCH}_{2} \mathrm{O}$ & 101.11 & - & - & 100.99 & - \\
\hline \multicolumn{6}{|l|}{$\mathrm{CH}_{3}$} \\
\hline MeO-6 & 56.38 & $56.50(55.85)$ & 56.38 & 55.66 & 56.25 \\
\hline MeO-7 & 56.31 & $56.16(56.28)$ & 56.24 & 55.66 & 56.06 \\
\hline MeO-2' & - & - & - & 55.91 & 60.68 \\
\hline MeO-3' & - & $55.62(55.64)$ & 55.85 & - & 60.53 \\
\hline MeO-4' & - & $56.02(55.64)$ & - & - & 55.76 \\
\hline
\end{tabular}

* The multiplicity of the signals was deduced by comparative analysis of the PND - and DEPT- ${ }^{13} \mathrm{C}$ NMR spectra. ${ }^{\mathrm{a}}$ The heteronuclear $2 \mathrm{D}$ shift-correlated via one bond $\left({ }^{1} \mathrm{Hx}{ }^{13} \mathrm{C}-\mathrm{COSY}-{ }^{1} \mathrm{~J}_{\mathrm{CH}}\right)$ and long-range couplings $\left({ }^{1} \mathrm{Hx}^{13} \mathrm{C}-\mathrm{COSY}-{ }_{-}{ }^{\mathrm{J}} \mathrm{CH}, \mathrm{n}=2\right.$ and 3 ) spectra (Table 3 ) were also used for these assignments. 
of $2,3 \mathbf{3}, 5$ and 7, and consequently of the other isoflavonoids isolated from Pterodon polygalaeflorus by comparison with data now unambiguously assigned (Tables 1-4).
Thus, a series of 2D NMR experiments led to the assignment of all ${ }^{1} \mathrm{H}$ - and ${ }^{13} \mathrm{C}$ resonances for $\mathbf{2}$ and $\mathbf{3 a}(e$. g.). In the ${ }^{1} \mathrm{H}-\mathrm{x}{ }^{13} \mathrm{C}-\mathrm{COSY}-{ }^{1} \mathrm{~J}_{\mathrm{CH}}$ spectra of $\mathbf{2}$ and $\mathbf{3 a}$ the connectivities between the protonated carbon atoms and

Table 2. ${ }^{1} \mathrm{H}$ - NMR data for the isoflavonoids 1-7 [ $200 \mathrm{MHz}(\mathbf{1}, \mathbf{2}, \mathbf{3 a}, \mathbf{5}$ and 7), $100 \mathrm{MHz}(\mathbf{6})$ and $60 \mathrm{MHz}(\mathbf{4 a})]$, in $\mathrm{CDCl}_{3}$ and TMS as internal standard, chemical shifts in $\delta(\mathrm{ppm})$ and coupling constants ( $\mathrm{J}$, in parenthesis) in Hz.*

\begin{tabular}{|c|c|c|c|c|c|c|c|}
\hline $\mathbf{H}$ & 1 & 2 & $3 a$ & $4 a$ & 5 & 6 & 7 \\
\hline 2 & $7.91(s)$ & $7.97(s)$ & $7.97(s)$ & $7.94(s)$ & $7.88(s)$ & $8.05(s)$ & $7.93(s)$ \\
\hline 5 & $7.61(s)$ & $7.63(s)$ & $7.60(s)$ & $7.74(s)$ & $7.59(s)$ & $7.67(s)$ & $7.63(s)$ \\
\hline 8 & $6.86(s)$ & $6.88(s)$ & $6.87(s)$ & $7.22(s)$ & $6.85(s)$ & $6.93(s)$ & $6.89(s)$ \\
\hline $2^{\prime}$ & $7.10(d, 1.7)$ & $7.25(d, 1.8)$ & $7.34(d, 1.6)$ & $7.1-6.7(m)$ & - & - & - \\
\hline $3^{\prime}$ & - & - & - & - & $6.59(s)$ & $6.68(s)$ & - \\
\hline 5 & $6.85(d, 8.0)$ & $6.93(d, 8.3)$ & $7.07(d, 8.2)$ & $7.1-6.7(m)$ & - & - & $6.76(d, 8.5)$ \\
\hline $6^{\prime}$ & $6.98(d d, 8.0,1.7)$ & $7.05(d d, 8.3,1.8)$ & $7.00(d d, 8.2,1.6)$ & $7.1-6.7(m)$ & $6.80(s)$ & $7.02(s)$ & $7.06(d, 8.5)$ \\
\hline $\mathrm{OCH}_{2} \mathrm{O}$ & $5.98(s)$ & - & - & $6.00(s)$ & $5.93(s)$ & $4.00(s)$ & - \\
\hline $\mathrm{MeO}-6$ & $3.98(s)$ & $3.99(s)$ & $3.97(s)$ & $3.95(s)$ & $3.96(s)$ & $4.00(s)$ & $4.01(s)$ \\
\hline $\mathrm{MeO}-7$ & $3.98(s)$ & $3.99(s)$ & $3.98(s)$ & - & $3.96(s)$ & $4.00(s)$ & $4.00(s)$ \\
\hline MeO-2' & - & - & - & - & $3.70(s)$ & $3.80(s)$ & $3.84(s)$ \\
\hline $\mathrm{MeO}-3$, & - & $3.92(s)$ & $3.86(s)$ & - & - & - & $3.95(s)$ \\
\hline $\mathrm{MeO}-4$ ' & - & $3.88(s)$ & - & - & - & $3.88(s)$ & $3.91(s)$ \\
\hline $\mathrm{MeO}-5$ & - & - & - & - & - & $3.97(s)$ & - \\
\hline AcO-4' & - & - & $2.32(s)$ & - & - & - & - \\
\hline AcO-7' & - & - & - & $2.40(s)$ & - & - & - \\
\hline
\end{tabular}

*Homonuclear ${ }^{1} \mathrm{Hx}{ }^{1} \mathrm{H}-\mathrm{COSY}$ and heteronuclear ${ }^{1} \mathrm{Hx}{ }^{13} \mathrm{C}-\mathrm{COSY}-{ }^{1} \mathrm{~J}_{\mathrm{CH}} 2 \mathrm{D}$ NMR spectra were also used for these assignments. Chemical shifts and coupling constants (J) were obtained from $1 \mathrm{D}{ }^{1} \mathrm{H}-\mathrm{NMR}$ spectra.

Table 3. Heteronuclear 2D shift-correlated via long-range couplings $\left[{ }^{1} \mathrm{H}-\mathrm{x}^{13} \mathrm{C}-\mathrm{COSY}-{ }^{\mathrm{n}} \mathrm{JCH}_{\mathrm{CH}}(\mathrm{n}=2\right.$ and 3), COLOC $]$ NMR spectral data for the isoflavonoids $\mathbf{2}, \mathbf{3 a}, \mathbf{5}$ and 7 .*

\begin{tabular}{|c|c|c|c|c|c|c|c|}
\hline \multicolumn{2}{|r|}{2} & \multicolumn{2}{|c|}{$3 a$} & \multicolumn{2}{|r|}{5} & \multicolumn{2}{|c|}{7} \\
\hline $\mathrm{C}^{2} \mathrm{~J}_{\mathrm{CH}}$ & ${ }^{3} \mathrm{~J}_{\mathrm{CH}}$ & ${ }^{2} \mathrm{~J}_{\mathrm{CH}}$ & ${ }^{3} \mathrm{~J}_{\mathrm{CH}}$ & ${ }^{2} \mathrm{~J}_{\mathrm{CH}}$ & ${ }^{3} \mathrm{~J}_{\mathrm{CH}}$ & ${ }^{2} \mathrm{~J}_{\mathrm{CH}}$ & ${ }^{3} \mathrm{~J}_{\mathrm{CH}}$ \\
\hline $3 \mathrm{H}-2$ & H-2'; H-6' & H-2' & H-2' & & H-6 & $\mathrm{H}-2$ & H-6' \\
\hline 4 & H-2; H-5 & & H-2; H-5 & & H-5 & & H-2; H-5 \\
\hline $6 \mathrm{H}-5$ & H-8; MeO-6 & & H-8; MeO-6 & & H-8; MeO-6 & & H-8; MeO-6 \\
\hline $7 \mathrm{H}-8$ & $\mathrm{H}-5 ; \mathrm{MeO}-7$ & $\mathrm{H}-8$ & H-5; MeO-7 & & $\mathrm{H}-5 ; \mathrm{MeO}-7$ & & H-5; MeO-7 \\
\hline $9 \mathrm{H}-8$ & H-2; H-5 & H-8 & H-5 & & H-5 & $\mathrm{H}-8$ & H-2; H-5 \\
\hline $10 \mathrm{H}-5$ & $\mathrm{H}-8$ & H-5 & & & $\mathrm{H}-8$ & & $\mathrm{H}-8$ \\
\hline $1^{\prime}$ & H-2; H-5' & & H-2; H-5' & & H-3' & & $\mathrm{H}-5$ \\
\hline $2^{\prime}$ & & & H-6’ & & $\mathrm{MeO}-2$ ' & & H-6'; MeO-2' \\
\hline $3^{\prime}$ & H-5'; MeO-3' & & H-5'; MeO-3' & & & & H-5'; MeO-3' \\
\hline 4 & $\mathrm{H}-2$ '; H-6'; MeO-4' & & H-2'; H-6' & & H-6 & & H-6'; MeO-4' \\
\hline 5 & & & & & H-3' & & \\
\hline $6^{\prime}$ & & & H-2' & & & & \\
\hline
\end{tabular}

* The chemical shifts for each one of the carbon and hydrogen atoms are described in Tables 1 and 2, respectively. The corresponding 2D NMR spectra were obtained a Bruker AC-200 $\left({ }^{1} \mathrm{H}: 200 \mathrm{MHz} ;{ }^{13} \mathrm{C}: 50.3 \mathrm{MHz}\right)$. 
the corresponding hydrogens that were observed are: $\mathrm{CH}-2$ [2: $\delta_{\mathrm{C}} 151.77(d)$ and $\delta_{\mathrm{H}} 7.97(s) ; 3 \mathbf{3 a} \delta_{\mathrm{C}} 152.49(d)$ and $\delta_{\mathrm{H}}$ $7.97(s)$ ], CH-5 [2: $\delta_{\mathrm{C}} 104.35(d)$ and $\delta_{\mathrm{H}} 7.63(s) ; 3 \mathbf{3 a}: \delta_{\mathrm{C}}$ $104.60(d)$ and $\delta_{\mathrm{H}} 7.60(s)$ ], CH-8 [2: $\delta_{\mathrm{C}} 99.18(d)$ and $\delta_{\mathrm{H}}$ $6.88(s)$; 3a: $\delta_{\mathrm{C}} 99.41(d)$ and $\delta_{\mathrm{H}} 6.87(s)$ ], CH-2' [2: $\delta_{\mathrm{C}}$ $112.13(d)$ and $\delta_{\mathrm{H}} 7.25(d, \mathrm{~J}=1.8 \mathrm{~Hz}) ; 3 \mathrm{a}: \delta_{\mathrm{C}} 113.55(d)$ and $\delta_{\mathrm{H}} 7.34(d, \mathrm{~J}=1.6 \mathrm{~Hz})$ ], $\mathrm{CH}-5$ ' [2: $\delta_{\mathrm{C}} 110.75(d)$ and $\delta_{\mathrm{H}} 6.93(d, \mathrm{~J}=8.3 \mathrm{~Hz}) ; 3 \mathrm{a}: \delta_{\mathrm{C}} 122.80(d)$ and $\delta_{\mathrm{H}} 7.07(d, \mathrm{~J}$ $=8.2 \mathrm{~Hz}$ ); shifted downfield by only $0.14 \mathrm{ppm}\left[\Delta \delta_{\mathrm{H}}=7.07\right.$ (3a)-6.93 (2)] and by $12.05 \mathrm{ppm}\left[\Delta \delta_{\mathrm{C}}=122.80(\mathbf{3 a})-110.75\right.$ (2) in the acetyl derivative (3a), as anticipated by shielding reduction of the mesomeric_ortho-effect], CH-6' [2: $\delta_{\mathrm{C}}$ $120.61(d)$ and $\delta_{\mathrm{H}} 7.05(d d, \mathrm{~J}=8.3$ and $\mathrm{J}=1.8 \mathrm{~Hz}) ; 3 \mathrm{a}: \delta_{\mathrm{C}}$ $120.66(d)$ and $\delta_{\mathrm{H}} 7.00(d d, \mathrm{~J}=8.2$ and $\left.\mathrm{J}=1.6 \mathrm{~Hz})\right]$ and methoxy groups [2: $\delta_{\mathrm{C}} 56.50$ and $\delta_{\mathrm{H}} 3.99,56.16$ and 3.99 , 55.62 and $3.92,56.02$ and $3.88 ; 3 \mathbf{3 a}: \delta_{\mathrm{C}} 56.38$ and $\delta_{\mathrm{H}} 3.87$, 56.24 and 3.98, 55.85 and 3.86] (Tables 1 and 2).
The chemical shift assignments of the quaternary carbon atoms were established by ${ }^{1} \mathrm{H}-\mathrm{x}^{13} \mathrm{C}-\mathrm{COSY}{ }^{n} \mathrm{~J}_{\mathrm{CH}}(\mathrm{n}=2$ and 3, COLOC) spectra. Thus, the spectrum of 2 showed long-range correlations (Table 3$)$ : $\mathrm{C}-3\left(\delta_{\mathrm{C}} 123.91\right)$ with $\mathrm{H}-2$ $\left(\delta_{\mathrm{H}} 7.97,{ }^{2} \mathrm{~J}_{\mathrm{CH}}\right), \mathrm{H}-2{ }^{\prime}\left(\delta_{\mathrm{H}} 7.25,{ }^{3} \mathrm{~J}_{\mathrm{CH}}\right)$ and H-6' $\left(\delta_{\mathrm{H}} 7.05\right.$, $\left.{ }^{3} \mathrm{~J}_{\mathrm{CH}}\right)$; C-4 with H-2 $\left(\delta_{\mathrm{H}} 7.97,{ }^{3} \mathrm{~J}_{\mathrm{CH}}\right)$ and $\mathrm{H}-5\left(\delta_{\mathrm{H}} 7.63,{ }^{3} \mathrm{~J}_{\mathrm{CH}}\right)$; C-6 $\left(\delta_{\mathrm{C}} 147.36\right)$ with H-5 $\left(\delta_{\mathrm{H}} 7.63,{ }^{2} \mathrm{~J}_{\mathrm{CH}}\right), \mathrm{H}-8\left(\delta_{\mathrm{H}} 6.88\right.$, $\left.{ }^{3} \mathrm{~J}_{\mathrm{CH}}\right)$ and MeO-6 $\left(\delta_{\mathrm{H}} 3.99,{ }^{3} \mathrm{~J}_{\mathrm{CH}}\right)$; C-7 $\left(\delta_{\mathrm{C}} 154.02\right)$ with H-8 $\left(\delta_{\mathrm{H}} 6.88,{ }^{2} \mathrm{~J}_{\mathrm{CH}}\right), \mathrm{H}-5\left(\delta_{\mathrm{H}} 7.63,{ }^{3} \mathrm{~J}_{\mathrm{CH}}\right)$ and MeO-7 $\left(\delta_{\mathrm{H}} 3.99\right.$, $\left.{ }^{3} \mathrm{~J}_{\mathrm{CH}}\right) ; \mathrm{C}-9\left(\delta_{\mathrm{C}} 151.89\right)$ with $\mathrm{H}-8\left(\delta_{\mathrm{H}} 6.88,{ }^{2} \mathrm{~J}_{\mathrm{CH}}\right), \mathrm{H}-2\left(\delta_{\mathrm{H}}\right.$ $\left.7.97,{ }^{3} \mathrm{~J}_{\mathrm{CH}}\right)$ and $\mathrm{H}-5\left(\delta_{\mathrm{H}} 7.63,{ }^{3} \mathrm{~J}_{\mathrm{CH}}\right) ; \mathrm{C}-10\left(\delta_{\mathrm{C}} 177.51\right)$ with H-5 $\left(\delta_{\mathrm{H}} 7.63,{ }^{2} \mathrm{~J}_{\mathrm{CH}}\right)$ and H-8 $\left(\delta_{\mathrm{H}} 6.88,{ }^{3} \mathrm{~J}_{\mathrm{CH}}\right) ; \mathrm{C}-1$ ' $\left(\delta_{\mathrm{c}}\right.$ $124.51)$ with $\mathrm{H}-2\left(\delta_{\mathrm{H}} 7.97,{ }^{3} \mathrm{~J}_{\mathrm{CH}}\right)$ and $\mathrm{H}-5,\left(\delta_{\mathrm{H}} 6.93,{ }^{3} \mathrm{~J}_{\mathrm{CH}}\right)$; C-3' $\left(\delta_{\mathrm{C}} 148.33\right)$ with $\mathrm{H}-5{ }^{\prime}\left(\delta_{\mathrm{H}} 6.93,{ }^{3} \mathrm{~J}_{\mathrm{CH}}\right)$ and MeO-3' $\left(\delta_{\mathrm{H}}\right.$ $\left.3.92,{ }^{3} \mathrm{~J}_{\mathrm{CH}}\right)$; C-4' $\left(\delta_{\mathrm{C}} 148.65\right)$ with H-2' $\left(\delta_{\mathrm{H}} 7.25,{ }^{3} \mathrm{~J}_{\mathrm{CH}}\right), \mathrm{H}-6$ $\left(\delta_{\mathrm{H}} 7.05,{ }^{3} \mathrm{~J}_{\mathrm{CH}}\right)$ and $\mathrm{MeO}-4{ }^{\prime}\left(\delta_{\mathrm{H}} 3.88,{ }^{3} \mathrm{~J}_{\mathrm{CH}}\right)$. The spectra of 3a, 5 and $7\left(\delta_{\mathrm{H}} 7.97,{ }^{3} \mathrm{~J}_{\mathrm{CH}}\right)$ revealed analogous results as shown in Table 3.

Table 4. NOE difference spectra $\left({ }^{1} \mathrm{H}-\left\{{ }^{1} \mathrm{H}\right\}-\mathrm{NOE}\right)$ data for the isoflavonoids $\mathbf{1}, \mathbf{2}, \mathbf{3 a}, \mathbf{5}$ and $\mathbf{7}$.

\begin{tabular}{|c|c|c|c|c|c|}
\hline \multirow[b]{2}{*}{ Compound } & \multicolumn{2}{|c|}{$\mathrm{H}$} & \multicolumn{3}{|c|}{ NOE enhancements } \\
\hline & H & $\delta_{\mathbf{H}}$ & H & $\delta_{\mathbf{H}}$ & $\%$ \\
\hline \multirow[t]{5}{*}{1} & $(\mathrm{MeO})_{2}-6,7$ & 3.98 & 5 & 7.61 & 5 \\
\hline & & & 8 & 6.86 & 10 \\
\hline & 5 & 7.61 & $\mathrm{MeO}-6$ & 3.98 & 15 \\
\hline & 2 & 7.91 & $2^{\prime}$ & 7.10 & 7 \\
\hline & & & 6 & 6.98 & 10 \\
\hline \multirow[t]{4}{*}{2} & MeO-4' & 3.88 & 5 , & 6.93 & 6 \\
\hline & MeO-3' & 3.92 & $2^{\prime}$ & 7.25 & 8 \\
\hline & $(\mathrm{MeO})_{2}-6,7$ & 3.99 & 5 & 7.63 & 6 \\
\hline & & & 8 & 6.88 & 5 \\
\hline \multirow[t]{3}{*}{$\mathbf{3 a}$} & $\mathrm{MeO}-3$ & 3.86 & $2^{\prime}$ & 7.34 & 9 \\
\hline & $(\mathrm{MeO})_{2}-6,7$ & $3.97,3.98$ & 5 & 7.60 & 6 \\
\hline & & & 8 & 6.87 & 6 \\
\hline \multirow[t]{4}{*}{5} & $\mathrm{MeO}-2$ & 3.70 & $3^{\prime}$ & 6.59 & 11 \\
\hline & & & 2 & 7.88 & $>1$ \\
\hline & $2 \mathrm{MeO}-6,7$ & 3.96 & 5 & 7.59 & 4 \\
\hline & & & 8 & 6.85 & 12 \\
\hline \multirow[t]{4}{*}{7} & $\mathrm{MeO}-2$ & 3.84 & 2 & 7.93 & 5 \\
\hline & MeO-4' & 3.91 & 5 , & 6.76 & 7 \\
\hline & $2 \mathrm{MeO}-6,7$ & $4.00,4.01$ & 5 & 7.63 & 7 \\
\hline & & & 8 & 6.89 & 9 \\
\hline
\end{tabular}


Homonuclear NOE difference $\left({ }^{1} \mathrm{Hx}\left\{{ }^{1} \mathrm{H}\right\}\right.$-NOE) spectra (Table 4) of compounds 1, 2, 3a, $\mathbf{5}$ and $\mathbf{7}$ contributed to the assignments (Tables 1 and 2). The EIMS and IR spectra were also used (vide experimental).

\section{Experimental}

\section{General experimental procedures}

Mps were determined on a Mettler PF-5 melting point analyser. IR spectra were recorded in $\mathrm{KBr}$ using a PerkinElmer 720 infrared spectrometer. UV spectra were recorded in MeOH on a Varian-UV/ VIS 634-5 spectrometer. ${ }^{1} \mathrm{H}$ - and ${ }^{13} \mathrm{C}-\mathrm{NMR}$ spectra were obtained on a Bruker AC-200 spectrometer with standard pulse sequences operating at $200 \mathrm{MHz}$ and $50.3 \mathrm{MHz}$, respectively, except the ${ }^{1} \mathrm{H}-\mathrm{NMR}$ of $\mathbf{4 a}$ and $\mathbf{6}$ which were recorded on a Varian EM-360 (60 MHz) and Varian XL-100 spectrometers, respectively. The chemical shift values are reported in $\delta$ (ppm) and the coupling constants ( $\mathrm{J}$ ) are in $\mathrm{Hz}$; carbon multiplicities were determined by DEPT experiments; ${ }^{1} \mathrm{H}$ $\mathrm{x}{ }^{1} \mathrm{H}-\mathrm{COSY},{ }^{1} \mathrm{H}-\mathrm{x}{ }^{13} \mathrm{C}-\mathrm{COSY}-{ }^{1} \mathrm{~J}_{\mathrm{CH}},{ }^{1} \mathrm{H}-\mathrm{x}{ }^{13} \mathrm{C}-\mathrm{COSY}$ $-{ }^{n} J_{C H}(n=2$ and 3, COLOC), NOE difference spectra NMR experiments were carried out using Bruker commercial microprograms. Low-resolution EIMS $(70 \mathrm{eV})$ data were obtained on a GC/MS Finningan 3300F/ 9500 apparatus. Chromatography was performed using Merck Kieselgel 0.05-0.20 mesh and TLC with Merck Kieselgel $60 \mathrm{~F}_{254}$. TLC plates were examined under UV illumination and after exposure to iodine vapour.

\section{Plant material}

A specimen of Pterodon polygalaeflorus was collected in Monte Alegre - Bom Jesus, Piauí State, Brazil and identified by Professor Afrânio Gomes Fernandes (Universidade Federal do Ceará, Fortaleza, Ceará, Brazil). A voucher specimen is deposited at the Herbarium Prisco

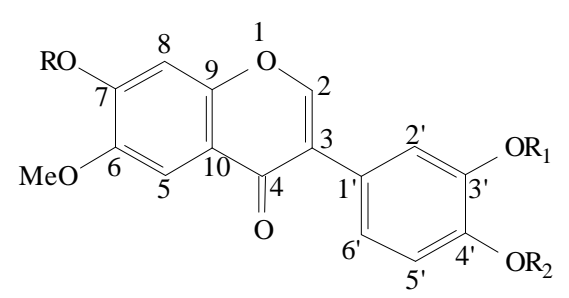

\begin{tabular}{lcccr} 
& $\mathrm{R}$ & $\mathrm{R}$ & $\mathrm{R}$ \\
$\mathbf{1}$ & $\mathrm{Me}$ & \multicolumn{2}{c}{$-\mathrm{CH}_{2}-r$} \\
$\mathbf{2}$ & $\mathrm{Me}$ & $\mathrm{Me}$ & $\mathrm{Me}$ \\
$\mathbf{3}$ & $\mathrm{Me}$ & $\mathrm{Me}$ & $\mathrm{H}$ \\
$\mathbf{3 a}$ & $\mathrm{Me}$ & $\mathrm{Me}$ & $\mathrm{Ac}$ \\
$\mathbf{4}$ & $\mathrm{H}$ & & $-\mathrm{CH}_{2}-$ \\
4a & $\mathrm{Ac}$ & & \multicolumn{2}{c}{$-\mathrm{CH}_{2}-$}
\end{tabular}

Bezerra of the Departamento de Biologia - Universidade Federal do Ceará.

\section{Isolation of pterodon polygalaeflorus constituents}

\section{Acetone extraction of the heartwood}

Dried and powdered heartwood $(4.4 \mathrm{Kg})$ was continuously extracted with hot acetone. Upon removal of the solvent a residue ( $189 \mathrm{~g}$ ) remained. This residue was chromatographed on a silica gel $(756 \mathrm{~g})$ column giving fractions $\mathrm{H}-1$ to $\mathrm{H}-5$, in this order, by elution with $n$-hexane- $\mathrm{CHCl}_{3}$ (1:1), $\mathrm{CHCl}_{3}, \mathrm{CHCl}_{3}$ - acetone (1:1), acetone and $\mathrm{MeOH}$. Fraction $\mathrm{H}-2$ (43 g, eluted with $\mathrm{CHCl}_{3}$ ) was rechromatographed on a silica gel column using cyclohexane- $\mathrm{CHCl}_{3}$ (1:1) and $\mathrm{CHCl}_{3}$-acetone $(8: 2,7: 3$ and 1:1) as eluents to obtain fraction $\mathrm{H}-2 \mathrm{a}$ to $\mathrm{H}-2 \mathrm{~d}$, respectively. Chromatography of fraction $\mathrm{H}-2 \mathrm{a}$ furnished $\mathbf{1}(56 \mathrm{mg}), \mathrm{H}-2 \mathrm{~b}$ afforded $\mathbf{1}$ (75 mg) and 7 (284 mg), H-2c yielded $\mathbf{1}$ (74 mg) and 2 (605 $\mathrm{mg}$ ) and $\mathrm{H}-2 \mathrm{~d}$ funished 2 (310 mg) and 5 (560 mg).

Fraction $\mathrm{H}-3$ (20 g), was eluted with $\mathrm{CHCl}_{3}$-acetone (1:1) and rechromatographed on a sílica gel column furnishing fractions $\mathrm{H}-3 \mathrm{a}$ to $3 \mathrm{c}$, in this order, by elution with $\mathrm{CHCl}_{3}$-EtOAc (9.5:0.05, 9:1 and 7.5:2.5). These fractions afforded $\mathbf{1}$ (61 mg), 2 (398 mg) and 5 (544 mg), 3 (200 mg), $6(283 \mathrm{mg})$ and $\mathbf{4}$ as acetyl derivative [4a (100 mg), obtained by treatment with $\mathrm{Ac}_{2} \mathrm{O} / \mathrm{Py}$, respectively, after rechromatographed on silica gel columns.

\section{Acetone extraction of the sapwood}

Dried and powdered sapwood $(4.4 \mathrm{Kg})$ was continuously extracted with hot acetone. The residue (200 g) obtained was chromatographed on a silica gel column using $\mathrm{CHCl}_{3}, \mathrm{CHCl}_{3}$-acetone (1:1), acetone and $\mathrm{MeOH}$ as eluents to furnish fractions 5-1 to 5-4. Fraction 5-1 (10 g) was rechromatographed on a sílica gel column to give $\mathbf{1}$ (50 $\mathrm{mg}), \mathbf{8}(20 \mathrm{mg}), \mathbf{9}(539 \mathrm{mg})$ and $\mathbf{1 0}(130 \mathrm{mg})$.<smiles>[R10]Oc1cc([R3])c(-c2coc3cc(OC)c(OC)cc3c2=O)c([R])c1OC</smiles>

$\begin{array}{ccccc} & \mathrm{R} & \mathrm{R}_{1} & \mathrm{R}_{2} & \mathrm{R}_{3} \\ \mathbf{5} & \mathrm{H} & & & \mathrm{OMe} \\ \mathbf{6} & \mathrm{H} & \mathrm{Me} & \mathrm{Me} & \mathrm{OMe} \\ \mathbf{7} & \mathrm{OMe} & \mathrm{Me} & \mathrm{Me} & \mathrm{H}\end{array}$


<smiles>COc1ccc(C(=O)O)cc1</smiles>

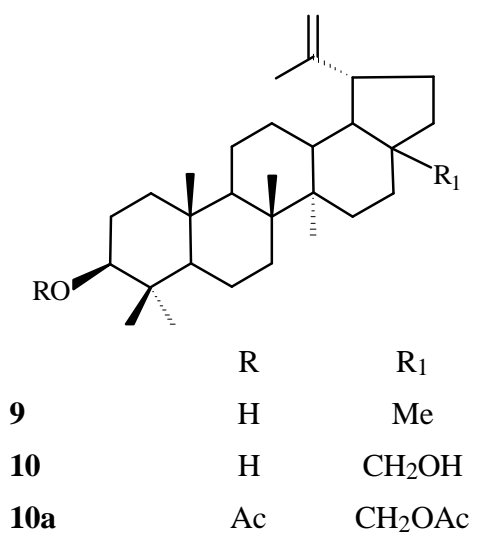

6,7-Dimethoxy-3',4'-methylenedioxyisoflavone (1)

Colorless crystals from MeOH, m.p. $240-241^{\circ} \mathrm{C}$. Spectral data are in accordance with values described in the literature $2 .{ }^{13} \mathrm{C}-\mathrm{NMR}$ : Table $1 .{ }^{1} \mathrm{H}-\mathrm{NMR}$ : Table 3. NOE difference spectra $\left({ }^{1} \mathrm{H}-\left\{{ }^{1} \mathrm{H}\right\}\right.$-NOE) data: Table 4.

3',4',6,7-Tetramethoxyisoflavone (2)

Colorless crystals from $\mathrm{MeOH}$, m.p. $188-189^{\circ} \mathrm{C}$. Spectral data are in accordance with literature values ${ }^{3,4} \cdot{ }^{13} \mathrm{C}$ NMR: Table 1. ${ }^{1} \mathrm{H}-\mathrm{NMR}$ : Table 2. Heteronuclear $2 \mathrm{D}{ }^{1} \mathrm{H}-$ $\mathrm{x}{ }^{13} \mathrm{C}$ shift-correlated via long-range coupling $\left({ }^{1} \mathrm{Hx}{ }^{13} \mathrm{C}\right.$ COSY ${ }^{n} J_{\mathrm{CH}}, \mathrm{n}=2$ and 3): Table 3. NOE difference spectra data: Table 4.

4-Hydroxy-3', 6,7-trimethoxyisoflavone (3)

Colorless crystals, m.p. $279-281^{\circ} \mathrm{C}$. IR $v_{\max } \mathrm{cm}^{-1}: 3240$ $(\mathrm{OH}), 1620(\mathrm{C}=\mathrm{O}), 1590,1510$ (aromatic). ${ }^{1} \mathrm{H}-\mathrm{NMR}$ (60 $\left.\mathrm{MHz}, \mathrm{CF}_{3} \mathrm{COOH}\right) \delta_{\mathrm{H}}: 8.80(s, \mathrm{H}-2), 7.84(s, \mathrm{H}-5), 7.50(s$, $\mathrm{H}-8), 7.10\left(m, \mathrm{H}-2^{\prime}, \mathrm{H}-5\right.$ ' and $\mathrm{H}-6$ '), 4.27( $\left.s, \mathrm{MeO}\right), 4.27$ ( $s$, $\mathrm{MeO})$ and $4.04(s, \mathrm{MeO})$. EIMS $m / z$ (rel. int.): 328 (100, $\left.[\mathrm{M}]^{+}\right), 313\left(6,[\mathrm{M}-\mathrm{Me}]^{+}\right), 285\left(5,[\mathrm{M}-\mathrm{Me}-\mathrm{CO}]^{+}\right), 181(8$, 3b), 180 (7, 3c), $148(19,3 d)$.

4'-O-Acetyl-3',6,7-trimethoxyisoflavone (3a)

Treatment of the isoflavone $3(100 \mathrm{mg})$ with $\left(\mathrm{Ac}_{2} \mathrm{O}\right)$ $(2 \mathrm{~mL})$ and pyridine $(2 \mathrm{~mL})$ at room temperature for $24 \mathrm{~h}$, and usual work-up, produced 3a $(98 \mathrm{mg})$, colorless crystals, m.p. 116-118 ${ }^{\circ} \mathrm{C}$. IR $v_{\max } \mathrm{cm}^{-1}: 1760$ (ester), $1625(\mathrm{C}=\mathrm{O})$, 1600, 1510 (aromatic). EIMS $m / z$ (rel. int.): $370\left(3,[\mathrm{M}]^{+}\right)$, $328\left(100,\left[\mathrm{M}-\mathrm{CH}_{2} \mathrm{C}=\mathrm{O}\right]^{++}\right), 327\left(20,[\mathrm{M}-\mathrm{Ac}]^{+}\right), 181(\mathbf{3}, \mathbf{3 b})$, $180(4,3 c)$. Heteronuclear $2 \mathrm{D}^{1} \mathrm{H}-\mathrm{x}{ }^{12} \mathrm{C}$ shift-correlated via long-range compling $\left({ }^{1} \mathrm{Hx}^{13} \mathrm{C}-\mathrm{COSY}-{ }^{\mathrm{n}} \mathrm{J}_{\mathrm{CH}}, \mathrm{n}=2\right.$ and 3): Table 3. ${ }^{13} \mathrm{C}-\mathrm{NMR}$ : Table 1. ${ }^{1} \mathrm{H}-\mathrm{NMR}$ : Table 2. NOE dfference spectra $\left({ }^{1} \mathrm{H}-\left\{{ }^{1} \mathrm{H}\right\}-\mathrm{NOE}\right)$ data: Table 4.

Methylation of $\mathbf{3}$

A solution of $\mathbf{3}(100 \mathrm{mg})$ in anhydrous acetone $(40 \mathrm{~mL})$ was treated with $\mathrm{Me}_{2} \mathrm{SO}_{4}(0.5 \mathrm{~mL})$ in the presence of calcinated $\mathrm{K}_{2} \mathrm{CO}_{3}$, under reflux during $24 \mathrm{~h}$. After filtration, the acetone was evaporated and the residue washed with $50 \% \mathrm{NH}_{4} \mathrm{OH}$. The remaining residue was crystallized from $\mathrm{MeOH}$ to give 2.

6-Methoxy-7-0-acetyl-3',4'-methylenedioxyisoflavone (4a)

Colorless crystals from $\mathrm{MeOH}$, m.p. $204-205{ }^{\circ} \mathrm{C}$. IR $v_{\max } \mathrm{cm}^{-1}: 1740$ (ester), $1650(\mathrm{C}=\mathrm{O}), 1610,1480$ (aromatic). EIMS $\boldsymbol{m} / \boldsymbol{z}$ (rel. int.) $354\left(42,[\mathrm{M}]^{+}\right), 312(100$, $\left.\left[\mathrm{M}-\mathrm{CH}_{2} \mathrm{C}=\mathrm{O}\right]^{+}\right), 311\left(20,[\mathrm{M}-\mathrm{Ac}]^{+}\right), 166(10,4 \mathrm{~b}), 146(18$, 4c). ${ }^{1} \mathrm{H}$ - NMR: Table 2.

2',6,7-Trimethoxy-4',5'-methylenedioxyisoflavone (5)

Colorless crystals from $\mathrm{CHCl}_{3}+\mathrm{MeOH}$, m.p. 237$239{ }^{\circ} \mathrm{C}$. Spectral data are in accordance with literature values ${ }^{2-4}{ }^{13} \mathrm{C}$-NMR: Table $1 .{ }^{1} \mathrm{H}-\mathrm{NMR}$ : Table 2 . Heteronuclear $2 \mathrm{D}^{1} \mathrm{H}-\mathrm{x}{ }^{13} \mathrm{C}$ shift-correlated via long-range coupling $\left({ }^{1} \mathrm{H} \mathrm{x}^{13} \mathrm{C}-\mathrm{COSY}-{ }^{\mathrm{n}} \mathrm{J}_{\mathrm{CH}}, \mathrm{n}=2\right.$ and 3): Table 3. NOE difference spectra $\left({ }^{1} \mathrm{H}-\left\{{ }^{1} \mathrm{H}\right\}-\mathrm{NOE}\right)$ : Table 4 .

\section{2',4',5',6,7-Pentamethoxyisoflavone (6)}

Colorless crystals from $\mathrm{MeOH}$, m.p. $169-172{ }^{\circ} \mathrm{C}$. Spectral data are in accordance with literature values ${ }^{5} .{ }^{1} \mathrm{H}$ NMR: Table 2.

\section{2',3',4',6,7- Pentamethoxyisoflavone (7)}

Colorless crystals, m.p. $170-172^{\circ} \mathrm{C}$. Spectral data are in accordance with literature values ${ }^{3.4} \cdot{ }^{13} \mathrm{C}-\mathrm{NMR}$ : Table 1 . ${ }^{1} \mathrm{H}-\mathrm{NMR}$ : Table 1. Heteronuclear ${ }^{1} \mathrm{H}-\mathrm{x}{ }^{13} \mathrm{C} 2 \mathrm{D}$ shift-correlated via long-range coupling $\left({ }^{1} \mathrm{Hx}^{13} \mathrm{C}-\mathrm{COSY}-{ }^{\mathrm{n}} \mathrm{J}_{\mathrm{CH}}, \mathrm{n}=\right.$ 2 and 3): Table 3. NOE difference spectra $\left({ }^{1} \mathrm{H}-\left\{{ }^{1} \mathrm{H}\right\}\right.$-NOE) data: Table 4.

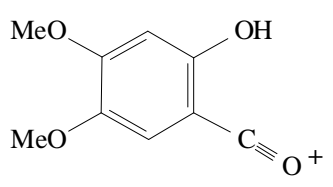

$\mathbf{3 b} m / z, 181$<smiles>[R9]C1=CC(=O)C(=C=O)C=C1OC</smiles>

3c $\mathrm{R}=\mathrm{Me}, m / z, 180$

4b $\mathrm{R}=\mathrm{H}, m / z, 166$

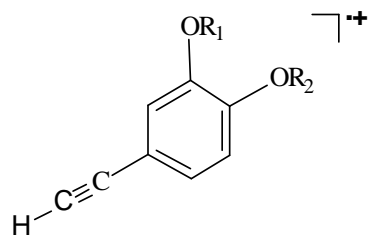

3d $\mathrm{R}_{1}=\mathrm{Me} \mathrm{R} \mathrm{R}_{2}=\mathrm{H}, m / z, 148$

4c $\mathrm{R}_{1}, \mathrm{R}_{2}=\mathrm{CH}_{2}, m / z, 146$ 
4-Methoxybenzoic acid (8, p-anisic acid)

Colorless crystals, m.p. $182-184{ }^{\circ} \mathrm{C}$. (Lit. ${ }^{10}$ m.p. $\left.184{ }^{\circ} \mathrm{C}\right) .{ }^{1} \mathrm{H}-\mathrm{NMR}\left(60 \mathrm{MHz}, \mathrm{CF}_{3} \mathrm{COOH}\right) \delta: 8.15(d, \mathrm{~J}=9.0$ $\mathrm{Hz}, 2 \mathrm{H}-2,6), 7.08(d, \mathrm{~J}=9.0 \mathrm{~Hz}, 2 \mathrm{H}-3,5), 4.02$ ( $s, \mathrm{MeO}-4)$. EIMS $m / z$ (rel. int.): $152\left(98,[\mathrm{M}]^{+}\right), 135\left(100,[\mathrm{M}-\mathrm{OH}]^{+}\right)$, 107 (13, [M-OH-CO and/or M-COOH $\left.]^{+}\right)$.

Lupeol (9)

Colorless crystals from $\mathrm{MeOH}$, m.p. $211-214^{\circ} \mathrm{C}$. [Lit. ${ }^{11}$ m.p. $215-216{ }^{\circ} \mathrm{C}\left(\mathrm{Me}_{2} \mathrm{CO}\right)$. Spectral data, mainly the chemical shifts and multiplicities of the signals of the carbon-13 deduced by comparative analysis of the PNDand DEPT- ${ }^{13} \mathrm{C}-\mathrm{NMR}$, and comparison with literature values $^{12}$ were used in the identification of this natural product.

\section{Betulin (10)}

Colorless crystals from MeOH, m.p. 249-251 ${ }^{\circ} \mathrm{C}$. $\left[\right.$ Lit. $^{13}$ m.p. $\left.251-252^{\circ} \mathrm{C}(\mathrm{EtOH})\right]$. Spectral data, mainly the chemical shifts and multiplicities of the signals of the carbon-13 deduced by comparative analysis of the PND- and DEPT${ }^{13} \mathrm{C}-\mathrm{NMR}$, including the diacetyl derivative (10a) and comparison with literature values ${ }^{12}$ were used in the identification of this compound.

\section{Acknowledgments}

This work was supported by CNPq fellowships and by grants from Conselho Nacional de Desenvolvimento Científico e Tecnológico (CNPq), Financiadora de Estudos e Projetos (FINEP), Coordenação de Aperfeiçoamento de Pessoal de Nível Superior (CAPES) and Programa de Apoio ao Desenvolvimento Científico e Tecnológico (PADCT). The authors are also grateful to Professor
Afrânio Gomes Fernandes, Universidade Federal do Ceará, for collection and identification of the plant material.

\section{References}

1.Campos, A.M.; Silveira, E.R.; Braz-Filho, R.; Teixeira, T.C. Phytochemistry 1994, 36, 403.

2. Ollis, W.D.; Rhodes, C.A.; Sutherland, I.O. Tetrahedron 1967, 23, 4741.

3. Braz-Filho, R.; Gottlieb, O.R.; Assumpção, R.M.V. An. Acad. Brasil. Ci. 1970, 42 (Supl.), 111.

4. Braz-Filho, R.; Gottlieb, O.R.; Assumpção, R.M.V. Phytochemistry 1971, 10, 2835.

5. Campbell, R.M.V.; Harper, S.H.; Kemp, A.D. J. Chem. Soc. C 1969, 1787.

6. Buckingham, J., Ed.; Dictionary of Organic Compounds; Chapman and Hall: London, 1982, 5th Edition (B-00951 and L-00498).

7. Tha, H.C.; Zilliken, F.; Breitmaier, E. Can. J. Chem. 1980, 58, 1211.

8. Sanders, J.K.M.; Hunter, B.K. In Modern NMR Spectroscopy: A Guide for Chemists; Oxford University Press; Oxford, 1993, 2nd Edition.

9. Breitmaier, E.; Voelter, W. In Carbon-13 NMR Spectroscopy

: High - Resolution Methods and Applications in Organic Chemistry and Biochemistry; VCH: Weinheim, 1987, 3rd Edition.

10. Reference 6: M-00529.

11. Reference 6: L-00498.

12. Mahato, S.B.; Kundu, A.P. Phytochemistry 1994, 37, 1517.

13. Reference 6: B-00951. 